\title{
Espaçamentos de plantio promovem produção distinta em híbrido de eucalipto
}

\author{
Robson Schaff Corrê $a^{1 *}$ Thelma Shirlen Soares ${ }^{1}$ Maiara Aparecida Alves ${ }^{1}$ Juliano Pereira de Sousa ${ }^{1}$ \\ Eduardo Morais Vieira ${ }^{1}$
}

${ }^{1}$ Universidade Federal de Jataí, Câmpus Jatobá, BR 364, km 195, no 3800, CEP 75801-615, Jataí, GO, Brasil

\begin{abstract}
Original Article
*Corresponding author: schaffcorrea@ufg.br

Keywords:

Eucalyptus

Aerial biomass

Planting density

Wood

Volumetric production

RESUMO: Este estudo objetivou avaliar a influência do espaçamento de plantio na produção volumétrica e de biomassa aérea de um híbrido de eucalipto, com 4,7 anos. O experimento foi conduzido empregando-se o delineamento em blocos casualizados, com três repetições e seis densidades de plantio de 1,5, 3,0, 4,5, 6,0, 7,5 e 9,0 $\mathrm{m}^{2}$ árvore $^{-1}$. Foi determinado o volume de madeira e de casca, a biomassa da madeira, casca, galhos e folhas para árvores e por área. A sobrevivência foi estimada com base no número de árvores vivas em relação ao número de árvores plantadas. As análises estatísticas foram realizadas por meio da ANOVA sendo procedido posteriormente análise de regressão. A sobrevivência das árvores não foi influenciada pelos espaçamentos de plantio. Foram observados valores crescentes da biomassa e volume por hectare com a redução do espaçamento de plantio. Maior produção de volume e biomassa por árvore foi obtida em espaçamentos mais amplos.
\end{abstract}

Palavras-chave:

Eucalyptus

Biomassa aérea

Densidade de plantio

Madeira

Produção volumétrica

Received in

2019/01/30

Accepted on

2020/07/03

Published in

2020/11/02

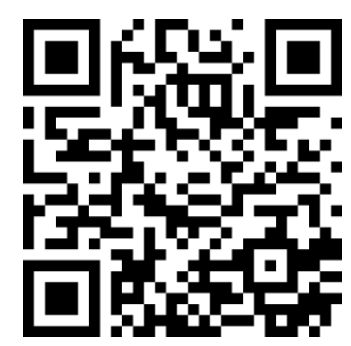

DOI: http://dx.doi.org/ 10.34062/afs.v7i3.7887

\section{Plant spacing promote distinct production in eucalypt hybrid}

\begin{abstract}
This study aimed was to evaluate the influence of planting spacing on the volumetric and aerial biomass production of a hybrid of eucalypt, with 4.7 years. The experiment was conducted using a randomized block design with three replications and six planting densities of 1,5, 3,0, 4,5, 6,0, 7,5 and 9,0 $\mathrm{m}^{2}$ tree $^{-1}$. The volume of wood and bark and biomass of wood, bark, twigs and leaves were determined. Survival was estimated based on the number of live trees in relation to the number of planted trees. Statistical analyzes were performed using ANOVA and regression analysis was performed. The survival of the trees was not influenced by the planting spacings. Increasing values of biomass and volume per hectare were observed with the reduction of planting spacing. The highest production of volume and biomass or individual trees was obtained at larger spacings.
\end{abstract}




\section{Corrêa et al.}

\section{Introdução}

O reflorestamento com eucalipto tem se tornado cada vez mais expressivo no Brasil em função da produção volumétrica obtida (Ribeiro et al. 2017), das potencialidades de uso da madeira serrada e laminada para movelaria e construção civil e como alternativa para suprir a crescente demanda de biomassa. Dessa forma, a silvicultura têm buscado identificar práticas silviculturais e técnicas de manejo que aumentem a produção de madeira por unidade de área para fins de geração de biomassa ou que agreguem qualidade ao produto final no caso da produção de madeira para finalidades nobres, como por exemplo serraria.

Entre os fatores condicionantes da produção florestal, o espaçamento exerce um dos papéis fundamentais no estabelecimento e condução de um povoamento, pois apresenta implicações do ponto de vista silvicultural, tecnológico e econômico, interferindo nas taxas de crescimento das plantas, na idade de corte, na qualidade da madeira, bem como nas práticas silviculturais empregadas e, consequentemente, nos custos de produção (Eloy et al. 2010).

Estudos com a finalidade de avaliar a produção em termos de volume e biomassa dos povoamentos florestais em diferentes espaçamentos de plantio tornam-se de grande importância para a implementação de práticas que propiciem o aumento da produtividade, diminuição de custos e/ou agregação de valor à árvores individuais.

Neste contexto também é importante a escolha do material genético a ser trabalhado, adotando-se parâmetros como situação frente ao registro de cultivares, compatibilidade com o clima e formato da parte área e radicular. Nestas condições optou-se pelo clone 1277, de domínio público. Visto que o clima onde o experimento está implantado apresenta menor propensão ao aparecimento de rachaduras de toras pelo crescimento mais lento, pois se observa transição da estação de crescimento vegetativo intenso para fraco ou moderado pela diminuição das chuvas (Paludzyszyn Filho e Santos, 2013). Além disto na condição de espaçamento adensado de plantio a utilização de material genético com área de copa reduzida pode favorecer $\mathrm{o}$ desenvolvimento das árvores.

Desta maneira o objetivo deste estudo foi avaliar a influência do espaçamento de plantio na produção volumétrica e de biomassa aérea de um híbrido de eucalipto, com 4,7 anos.

\section{Materiais e Métodos}

O estudo foi conduzido na Regional Jataí da Universidade Federal de Goiás localizada no município de Jataí - Estado de Goiás, sob coordenadas $17^{\circ} 56^{\prime} 10^{\prime \prime} \mathrm{S}$ e $51^{\circ} 43^{\prime} 40^{\prime \prime} \mathrm{O}$, com altitude de $662 \mathrm{~m}$. O clima da região, segundo a classificação de Köppen, é do tipo Aw, megatérmico, com a estação seca definida de maio a setembro, e a chuvosa, de outubro a abril. A temperatura média de $22{ }^{\circ} \mathrm{C}$ e precipitação média entre 1.200 a $1.800 \mathrm{~mm}$ anuais (INMET 2013)

O solo da área experimental, de acordo com o Sistema Brasileiro de Classificação de Solos, é classificado como Plintossolo (EMBRAPA 2013). A análise de solo da área experimental, cujas amostras foram coletadas a uma profundidade de 0 a $20 \mathrm{~cm}$ apresentou as seguintes características químicas e físicas: $\mathrm{pH}\left(\mathrm{CaCl}_{2}\right)=4,5 ; \mathrm{H}+\mathrm{Al}=6,2 \mathrm{cmol}_{\mathrm{c}} \mathrm{dm}^{-3}$; $\mathrm{Ca}=2,0 \mathrm{cmol}_{\mathrm{c}} \mathrm{dm}^{-3} ; \mathrm{Mg}=1,21 \mathrm{cmol}_{\mathrm{c}} \mathrm{dm}^{-3} ; \mathrm{Al}=$ $0,52 \mathrm{cmol}_{\mathrm{c}} \mathrm{dm}^{-3} ; \mathrm{K}=0,15 \mathrm{mg} \mathrm{dm}^{-3} ; \mathrm{P}=4,2 \mathrm{mg} \mathrm{dm}^{-}$ 3; $\mathrm{B}=0,04 \mathrm{mg} \mathrm{dm}^{-3} ; \mathrm{Cu}=8,2 \mathrm{mg} \mathrm{dm}^{-3} ; \mathrm{Zn}=2,1 \mathrm{mg}$ $\mathrm{dm}^{-3} ; \mathrm{S}=15 \mathrm{mg} \mathrm{dm}^{-3} ; \mathrm{Na}=3,1 \mathrm{mg} \mathrm{dm}^{-3} ; \mathrm{CTC}=9,6$ $\mathrm{cmol}_{\mathrm{c}} ;$ argila $=515 \mathrm{~g} \mathrm{dm}^{-3} ;$ silte $=75 \mathrm{~g} \mathrm{dm}^{-3}$ e areia $=410 \mathrm{~g} \mathrm{dm}^{-3}$.

O preparo do solo foi realizado por meio de aração e gradagem em área total, com posterior correção do solo com a aplicação de $400 \mathrm{~kg} \mathrm{ha}^{-1} \mathrm{de}$ calcário dolomítico, a adubação foi efetuada utilizando-se $100 \mathrm{~g}_{\text {planta }}{ }^{-1}$ de NPK $(2: 20: 18)+20 \mathrm{~g}$ planta $^{-1}$ de ureia e $20 \mathrm{~g}_{\text {planta }}{ }^{-1}$ de sulfato de boro.

$\mathrm{O}$ experimento foi implantado utilizando mudas de um híbrido de Eucalyptus grandis W. Hill. $\mathrm{x}$ Eucalyptus camaldulensis Dehnh. (clone 1277). Foram testadas seis distância entre plantas na linha de plantio, correspondendo às seguintes áreas disponíveis para crescimento: 1,$5 ; 3,0 ; 4,5 ; 6,0 ; 7,5$ e 9,0 $\mathrm{m}^{2}$ árvore $^{-1}$, correspondentes aos espaçamentos de plantio de $3,0 \mathrm{~m} \times 0,5 \mathrm{~m}, 3,0 \mathrm{~m} \times 1,0 \mathrm{~m}, 3,0 \mathrm{~m} \times$ $1,5 \mathrm{~m}, 3,0 \mathrm{~m} \times 2,0 \mathrm{~m}, 3,0 \mathrm{~m} \times 2,5 \mathrm{~m}$ e $3,0 \mathrm{~m} \times 3,0 \mathrm{~m}$, respectivamente. $\mathrm{O}$ delineamento experimental utilizado foi o de blocos casualizados, com três repetições e em cada uma das 18 unidades experimentais onde foram plantadas, além de bordadura simples, 18 mudas de eucalipto na área útil da parcela, divididas em três linhas de plantio.

Quando o povoamento estava com 4,7 anos, foi derrubada, em cada parcela, uma árvore de diâmetro próximo ao diâmetro médio da parcela, totalizando 18 árvores amostradas. As árvores foram abatidas a $10 \mathrm{~cm}$ do solo e os compartimentos foram separados em tronco (madeira e casca) e copa (galhos verdes e folhas).

Antes da derrubada foi efetuada uma avaliação do percentual de sobrevivência das árvores em cada espaçamento, a qual foi determinada com base na contagem das árvores vivas em relação ao número de árvores plantadas em cada parcela.

Determinou-se a massa de copa fresca de cada árvore utilizando-se uma balança de carga. Em campo foram retiradas amostras compostas de copa, acondicionadas em sacos plásticos para pesagem com balança analítica de precisão $( \pm 0,01 \mathrm{~g})$ para posterior acondicionamento em sacos de papel, secagem em estufa de circulação forçada de ar a 70 $\pm 5^{\circ} \mathrm{C}$ e pesagem, após se obter massa seca constante. A partir da relação entre a massa fresca e a massa seca das amostras determinou-se a biomassa 
de cada compartimento, em kg arvore ${ }^{-1}$, pela equação adaptada de Soares et al. (2011):

Biomassa $=[\mathrm{MF}$ x MSA $] / \mathrm{MFA} \quad$ [1]

Onde: biomassa $=$ massa seca do compartimento, em kg; MF = massa fresca do compartimento, em kg; MSA = massa seca da amostra, em kg; e MFA = massa fresca da amostra, em kg.

A biomassa do fuste, considerada até o diâmetro mínimo de $3,0 \mathrm{~cm}$, foi determinada multiplicando-se o volume pela densidade básica, da madeira e da casca, em cada tratamento.

Para a determinação do volume do fuste com e sem casca foi utilizada a cubagem rigorosa pelo método de Smalian medindo-se nas alturas $0,1 \mathrm{~m}$, 0,3 m, DAP (1,3 m do solo) e a cada $2,0 \mathrm{~m}$ de comprimento até um diâmetro-limite de $3,0 \mathrm{~cm}$. Para a determinação da densidade, foram retirados discos de madeira com casca de 3,0 - 5,0 cm de espessura correspondentes a $0 \%, 25 \%, 50 \%, 75 \%$ e $100 \%$ da altura comercial das árvores. Os discos foram utilizados para o cálculo da densidade básica da madeira e da casca, determinada pela norma NBR 11941-02 (ABNT 2003).

A biomassa da parte aérea e o volume foram estimados por hectare com base na sobrevivência de árvores nas parcelas. Sendo que a sobrevivência foi estimada com base no número de árvores plantadas em relação ao número de árvores vivas na época da avaliação.
Após a coleta dos dados, foi verificada a normalidade dos erros pelo teste de Shapiro-Wilk. No caso de não atendimento da distribuição normal, os dados foram transformados em $\sqrt{\mathrm{X}}$. Após esta etapa procedeu- se a análise de regressão ao nível de $5 \%$ de significância.

\section{Resultados e Discussão}

A taxa de sobrevivência aos 4,7 anos de idade não apresentou diferença estatística significativa entre tratamentos e variou de 64,9 a 71,2\% (Figura 1). Provavelmente, conforme relatam Ferreira et al. (2014), a pouca resposta de sobrevivência em função do espaçamento de plantio é provavelmente devida à boa qualidade do sítio. No presente estudo as taxas de sobrevivência são inferiores aos cerca de $95 \%$ encontrados por Reis et al. (2014) para diferentes clones, incluindo o clone 1277. Reis et al. (2014) não encontraram bom desempenho em um dos locais estudados, atribuindo este fato a problemas de instalação do plantio e/ou de especificidades do sítio, como por exemplo gradiente de fertilidade. Possivelmente algum fator de especificidade do sítio possa ter influenciado os resultados de sobrevivência do presente estudo já que não foi possível indicar a causa da baixa sobrevivência.

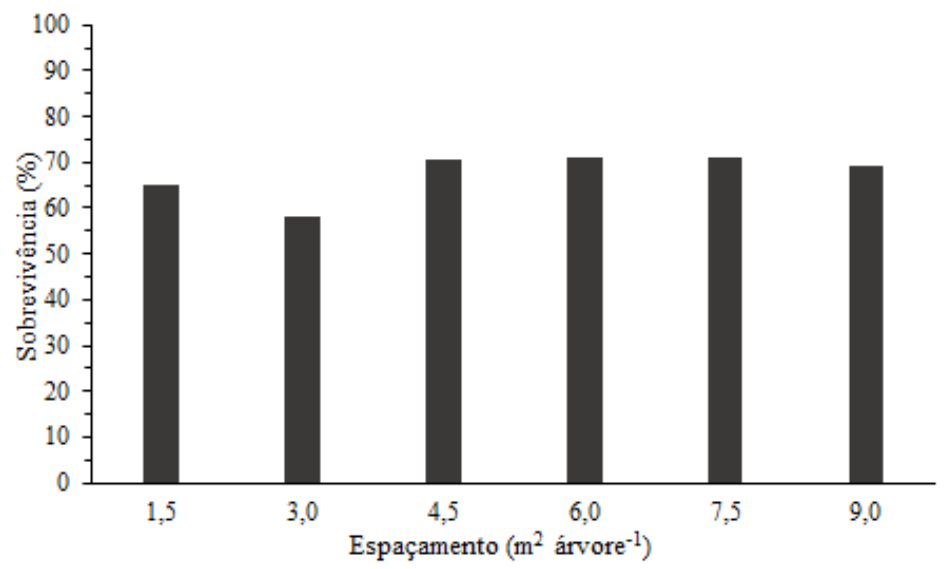

Figura 1. Sobrevivência de híbrido de eucalipto aos 4,7 anos em diferentes espaçamentos de plantios.

Não foi observada diferença estatística significativa entre os valores de densidade básica da madeira nos diferentes espaçamentos avaliados, mas o mesmo não ocorreu para a densidade básica da casca (Tabela 1). Para madeira os valores encontrados estão próximos aos de Santana et al. (2011) e para casca mostram-se intermediários considerando-se os trabalhos de Rocha et al. (2016) e Andrade et al. (2009). O efeito dos espaçamentos de plantio sobre a densidade básica da madeira também não foi encontrado por Sereghetti et al. (2015), como no presente estudo estes reportaram tendência de menor densidade em espaçamentos mais adensados. Para casca o comportamento foi o mesmo de Rocha et al. (2016), com maior densidade básica em menores densidades de plantio. Provavelmente isto ocorra pela maior proporção de lenho de maior idade sendo depositado nas árvores onde a estagnação do crescimento ainda não ocorreu, ou seja nos espaçamentos mais amplos. Este comportamento de aumento da densidade na direção medula-casca foi reportado por Mello et al. (2016). Tal comportamento deve ser levado em consideração em questões econômicas pois talvez em idade mais avançada a relação biomassa/volume seja mais vantajosa para questões de transporte. 


\section{Corrêa et al.}

Tabela 1. Valores médios de densidade básica da madeira e da casca de híbrido de eucalipto aos 4,7 anos em diferentes espaçamentos de plantio.

\begin{tabular}{ccc}
\hline Espaçamento & Densidade básica da madeira $\left(\mathbf{g ~ c m}^{-3}\right)$ & Densidade básica da casca $\left(\mathbf{g ~ c m}^{-3}\right)$ \\
\hline $3,0 \mathrm{~m} \times 0,5 \mathrm{~m}$ & $0,42 \mathrm{a}$ & $0,23 \mathrm{a}$ \\
$3,0 \mathrm{~m} \times 1,0 \mathrm{~m}$ & $0,43 \mathrm{a}$ & $0,26 \mathrm{~b}$ \\
$3,0 \mathrm{~m} \times 1,5 \mathrm{~m}$ & $0,43 \mathrm{a}$ & $0,26 \mathrm{~b}$ \\
$3,0 \mathrm{~m} \times 2,0 \mathrm{~m}$ & $0,44 \mathrm{a}$ & $0,26 \mathrm{~b}$ \\
$3,0 \mathrm{~m} \times 2,5 \mathrm{~m}$ & $0,44 \mathrm{a}$ & $0,28 \mathrm{~b}$ \\
$3,0 \mathrm{~m} \times 3,0 \mathrm{~m}$ & $0,45 \mathrm{a}$ & $0,28 \mathrm{~b}$ \\
\hline
\end{tabular}

Nota: médias seguidas por letras diferentes na coluna diferem estatisticamente pelo teste de Tukey $(\mathrm{p}<0,05)$.

As produções em volume e biomassa de cada espaçamento são apresentadas nas Figuras 2 e 3 , respectivamente.
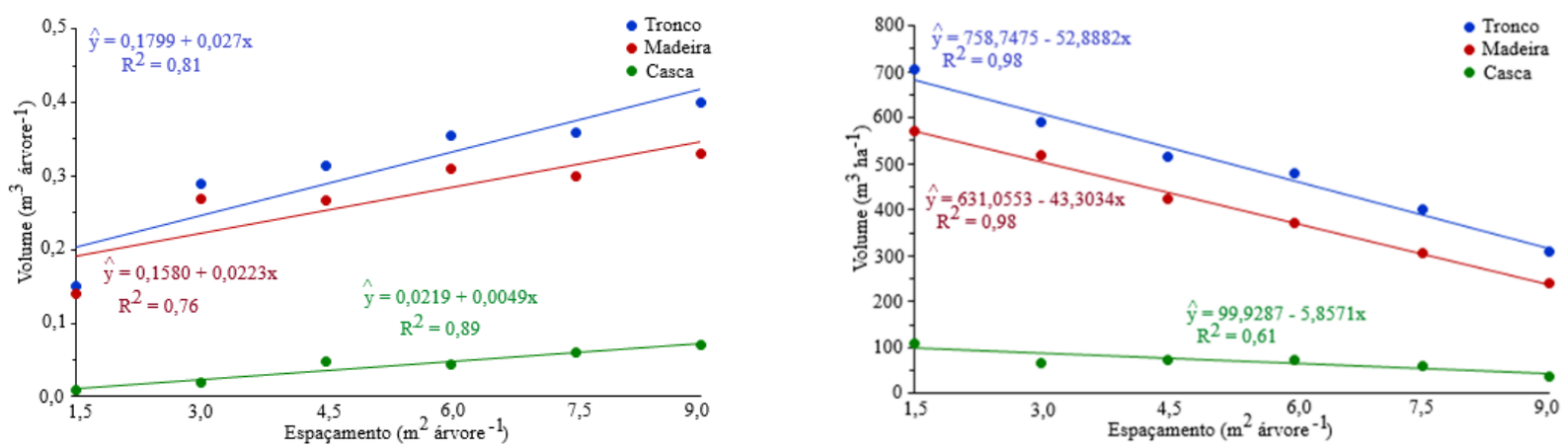

Figura 2. Produção volumétrica do tronco, madeira e casca (por árvore e por área) de híbrido de eucalipto aos 4,7 anos em diferentes espaçamentos de plantio.
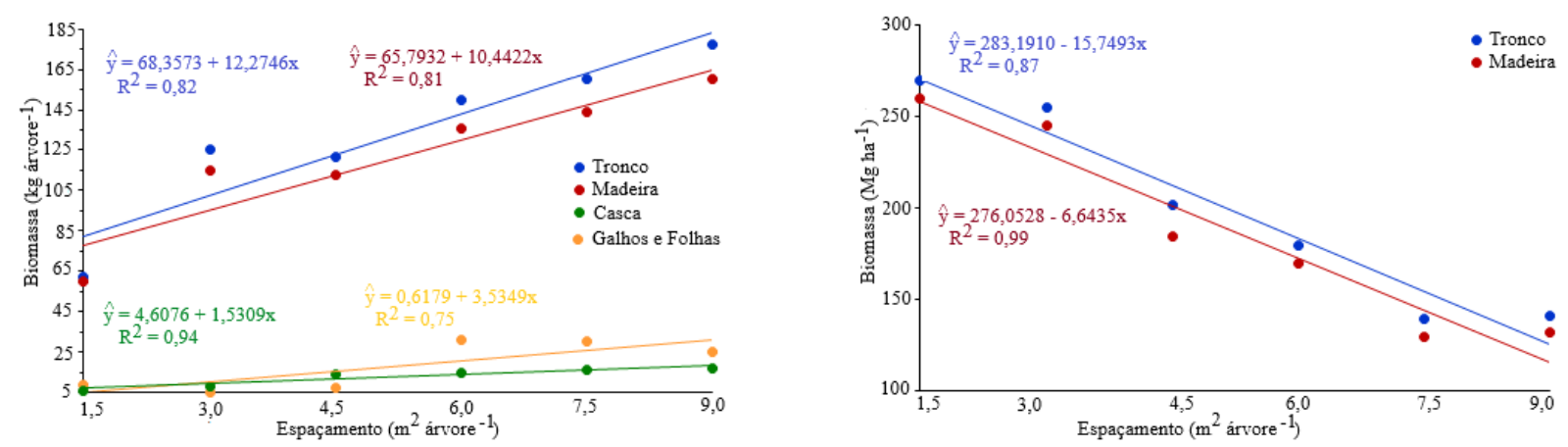

Figura 3. Produção de biomassa aérea de tronco, madeira (por árvore e por área), galhos e folhas e casca (por árvore e por área) de híbrido de eucalipto aos 4,7 anos em diferentes espaçamentos de plantio.

A maior produção volumétrica de madeira, casca e tronco $\left(\mathrm{m}^{3}\right.$ hectare $\left.^{-1}\right)$ foi obtida para $\mathrm{o}$ espaçamento mais adensado 1,5 $\mathrm{m}^{2}$ árvore $^{-1}(3,0 \mathrm{~m} \mathrm{x}$ $0,5 \mathrm{~m}$ ) em razão do maior número de indivíduos por unidade de área (Figura 2), decrescendo à medida que reduz a densidade de plantio. A diferença de produtividade volumétrica de madeira entre $\mathrm{O}$ espaçamento 3,0 m x 1,0 m com os demais (3,0 m x $1,5 \mathrm{~m} ; 3,0 \mathrm{~m} \times 2,0 \mathrm{~m}, 3,0 \mathrm{~m} \times 2,5 \mathrm{~m} \mathrm{e} \mathrm{3,0} \mathrm{m} \mathrm{x} \mathrm{3,0} \mathrm{m)}$ foi superior a 7\%, 28\%, 40\% e $53 \%$, respectivamente.
Estes resultados de aumento do volume de madeira com a diminuição do espaçamento corroboram com diversos autores (Oliveira et al. 2009, Ferreira et al. 2014, Sereghetti et al. 2015). Segundo Lima et al. (2013), altas densidades de plantio podem resultar em fustes mais tortuosos, pequenos e de diâmetros menores, ao passo que em espaçamentos maiores o espaço para o crescimento de cada árvore é ampliado, proporcionando melhor crescimento e desenvolvimento da planta, 
promovendo maiores diâmetros, maior conicidade e bom desenvolvimento radicular e da copa.

A diferença volumétrica do tronco entre o espaçamento mais produtivo $\left(1,5 \mathrm{~m}^{2}\right)$ e o menos produtivo $\left(9,0 \mathrm{~m}^{2}\right)$ foi cerca de $63,6 \%$, que corresponde a 448,15 $\mathrm{m}^{3} \mathrm{ha}^{-1}$. Passos et al. (2006) também constataram o aumento do volume do tronco com a menor densidade de árvores, segundo os mesmos autores o volume do tronco é diretamente proporcional ao número de árvores por área.

$\mathrm{O}$ volume de tronco, madeira e casca de árvore individual foi influenciado pelos espaçamentos, o espaçamento com a menor área por planta, de 1,5 $\mathrm{m}^{2} \mathrm{ha}^{-1}$, apresentou árvores com menor volume (Figura 2). Uma maior produção individual foi observada em espaçamentos mais amplos, como o de 9,0 $\mathrm{m}^{2}$ árvore $^{-1}$ e se deve a maior área útil por planta, resultando em maior rendimento de árvore individual (Reiner et al., 2011; Sereghetti et al. 2015), onde o espaço para o crescimento de cada árvore é ampliado, proporcionando melhor crescimento e desenvolvimento da planta, contribuindo diretamente na qualidade e na quantidade da produção em termos individuais (Lima et al., 2013).

Quanto menor o espaçamento maior é a produção de casca por hectare (Klein et al., 1992), a casca juntamente com a biomassa de galhos e folhas apresenta-se de extrema importância, pois podem ser utilizados para geração de energia (Santiago e Rezende, 2014), no entanto, há a contribuição destes compartimentos no processo de ciclagem de nutrientes, devolvendo ao solo macro e micronutrientes. Segundo Schumacher e Caldeira (2001) a casca pode alocar 26,40 kg ha-1, 280,30 kg $\mathrm{ha}^{-1}$ e $17,8 \mathrm{~kg} \mathrm{ha}^{-1}$ de $\mathrm{K}$, Ca e $\mathrm{Mg}$, respectivamente, contribuindo para a manutenção do estoque destes nutrientes minerais no solo, bem como para a produtividade de biomassa (Dick et al. 2016) caso permaneçam no local.

A produção de biomassa do tronco e de madeira por hectare (Figura 3) foi influenciada pelo espaçamento de plantio, o espaçamento mais adensado 1,5 $\mathrm{m}^{2}$ árvore ${ }^{1}$ gerou a maior produção de biomassa, e o mais amplo 9,0 $\mathrm{m}^{2}$ árvore ${ }^{-1}$ a menor, semelhante ao encontrado para a produção volumétrica $\left(\mathrm{m}^{3} \mathrm{ha}^{-1}\right)$. Para a produção de biomassa de tronco e madeira por árvore (Figura 3), os tratamentos com menores densidades de plantio apresentaram maior biomassa seca, resultados semelhantes a Müller et al. (2005).

Estes resultados corroboram com Caron et al. (2015) em que espaçamentos reduzidos resultaram em maior produção de biomassa. Freitas et al. (2004), em um povoamento de E. grandis aos nove anos de idade, obtiveram produção de biomassa de 142,31 Mg ha-1 em espaçamento 3,0 m x 2,0 m (6,0 $\mathrm{m}^{2}$ árvore $^{-1}$ ), ao passo em que Vieira et al. (2013) obtiveram 198,5 $\mathrm{Mg} \mathrm{ha}^{-1}$ de biomassa acima do solo em E. urophylla x E. globulus aos dez anos de idade.
No presente estudo, em 4,7 anos a produção de biomassa no espaçamento $3,0 \mathrm{~m} \times 0,5 \mathrm{~m}\left(1,5 \mathrm{~m}^{2}\right.$ árvore $^{-1}$ ) foi de $256,14 \mathrm{Mg} \mathrm{ha}^{-1}, 2,3$ e 1,2 vezes superior, respectivamente, com uma produção média $42 \%$ superior, mostrando que em espaçamentos mais densos pode-se obter maior produtividade e redução no tempo de corte, mas implicando em árvores de diâmetros inferiores.

Houve uma relação direta do volume $\left(\mathrm{m}^{3}\right.$ ha $\left.{ }^{1}\right)$ e biomassa $\left(\mathrm{Mg} \mathrm{ha}^{-1}\right)$ com a densidade de plantio, nos tratamentos com maiores densidades de plantio foram constatados os maiores volumes de madeira e quantidade de biomassa seca, resultados semelhantes a Müller et al. (2005).

A produção de biomassa de galhos verdes e secos e de folhas em unidade de área, não diferiu entre os diferentes espaçamentos de plantio, apresentando uma produção média de $19,18 \mathrm{Mg}$ ha 1 , essa semelhança entre os espaçamentos mais densos e mais amplos se deve principalmente ao maior número de árvores por hectare nos espaçamentos mais adensados, igualando a produção destes compartimentos, entretanto, nos espaçamentos mais amplos se tem a formação de galhos mais grossos. Resultados diferentes foram encontrados por Caron et al. (2015) em diferentes espécies florestais, onde a biomassa de galhos e de folhas decresceu com o aumento do espaçamento de plantio.

A biomassa de galhos verdes e secos e de folhas por árvore (Figura 3) variou entre os espaçamentos, aumentando gradativamente com a maior área útil por planta, onde os espaçamentos maiores apresentaram uma maior produção de folhas e galhos. Oliveira Neto et al. (2003), trabalhando com Eucalyptus camaldulensis aos 32 meses de idade, concluíram que os maiores valores de biomassa da parte aérea, por árvore, ocorreram em razão da reduzida competição entre plantas em densidades populacionais mais baixas.

A produção de biomassa de casca de árvore individual (Figura 3) apresentou um comportamento crescente, com uma diferença de 10,96 kg árvore ${ }^{-1}$ entre as áreas de 9,0 m² árvore ${ }^{-1}$ e 1,5 m² árvore ${ }^{-1}$, esta diferença ocorre pela maior competição por água, luz e nutrientes pelas plantas e menor área foliar dos espaçamentos mais densos. Já a biomassa de casca apresentou uma produção média de 19,64 $\mathrm{Mg} \mathrm{ha}^{-1}$.

A escolha do espaçamento ótimo seja para produção volumétrica ou de biomassa varia de acordo com a finalidade do plantio e o grau de mecanização das operações florestais (Oliveira Neto et al. 2010). Espaçamentos amplos geralmente são utilizados para fins de serraria e industrial, já os espaçamentos mais adensados, são utilizados para fins energéticos com ciclo de corte chegando a 5 anos (Silveira et al. 2014). Como exposto por Eufrade Junior et al. (2016) para espaçamentos adensados o método de corte pode ser adaptado com 
máquinas de corte de forragem para que se possa aproveitar a máxima produtividade por hectare destes espaçamentos. No entanto na colheita tradicional deve-se avaliar a produtividade e custo do sistema adotado em função do espaçamento de plantio (Leite et al., 2014).

\section{Conclusões}

O espaçamentos de plantio influencia a produção volumétrica e a biomassa aérea total por árvore individual. O espaçamento mais denso (3,0 m x $0,5 \mathrm{~m}$ ) proporciona maior produção volumétrica e de biomassa aérea por unidade de área, e os mais amplos por árvore individual.

\section{Agradecimentos}

Pela concessão de bolsas: M.A.A. à UFG (Universidade Federal de Goiás) e J.P.S. e E.M.V à CAPES (Coordenação de Aperfeiçoamento de Pessoal de Nível Superior).

\section{Referências Bibliográficas}

Andrade MCN, Minhoni MTA, Sansigolo CA, Zied DC. (2009) Densidade básica da madeira de sete espécies e três clones de eucalipto antes e durante o cultivo de shiitake. Acta Scientiarum. Agronomy, 31 (2): 235-240. doi: https://doi.org/10.1590/S180786212009000200008

Associação Brasileira de Normas Técnicas - ABNT. (2003) NBR 11941-02 - Determinação da densidade básica em madeira. Rio de Janeiro: ABNT. 6p.

Berger R, Schneider PR, Finger CAG, Haselein CR. (2002) Efeito do espaçamento e da adubação no crescimento de um clone de Eucalyptus saligna Smith. Ciência Florestal, 12 (2): 75-87.

Caron BO, Eloy E, Souza VQ, Schmidt D, Balbinot R, Behling A, Monteiro GC. (2015) Quantificação da biomassa florestal em plantios de curta rotação com diferentes espaçamentos. Comunicata Scientiae, 6 (1):106-112.

Dick G, Schumacher MV, Momolli DR, Santos JC, Ludvichak AA, Guimarães CC, Souza HP. (2016) Quantificação da biomassa e nutrientes em um povoamento de Eucalyptus dunnii Maiden estabelecido no Bioma Pampa. Revista Ecologia e Nutrição Florestal-ENFLO, 4 (1): 1-9. doi: http://dx.doi.org/10.5902/2316980X20589

Eloy E, Caron BO, Souza VQ, Trevisan R, Behling A, Bamberg R, Vian AL, Busanello C. (2010) Espécies florestais em plantios de curta rotação para biomassa. Revista da Madeira, 21: 50-53.

Empresa Brasileira de Pesquisa Agropecuária EMBRAPA. Centro Nacional de Pesquisa do Solo.
(2013) Sistema brasileiro de classificação de solos. 3 ed. Brasília: EMBRAPA. 353p.

Eufrade Junior HJ, Melo RX, Sartori MMP, Guerra SPS, Ballarin AW. (2016) Sustainable use of eucalypt biomass grown on short rotation coppice for bioenergy. Biomass and Bioenergy, 90: 15-21. doi:

https://doi.org/10.1016/j.biombioe.2016.03.037.

Ferreira DHAA, Leles PSL, Machado EC, Abreu AHM, Abilio FM. (2014) Crescimento de clone de Eucalyptus urophylla $x$ E. grandis em diferentes espaçamentos. Floresta, 44 (3): 431-440. doi: http://dx.doi.org/10.5380/rf.v44i3.32188.

Freitas R, Schumacher MV, Caldeira MVW, Spathelf P. (2004) Biomassa e conteúdo de nutrientes em povoamento de Eucalyptus grandis W. Hill ex Maiden plantado em solo sujeito à arenização, no município de Alegrete-RS. Biomassa \& Energia, 1 (1): 93-104.

Instituto Nacional de Meteorologia - INMET. (2013) BDMEP - Banco de Dados Meteorológicos para Ensino e Pesquisa: Série Histórica - Dados Diários de 01/01/1982 a 31/12/2012 Estação: 83464 - JATAI - GO. Disponível em: <http://www.inmet.gov.br/portal/index.php?r=bdme p/bdmep>. Acesso em: 11 dez. 2013.

Klein JEM, Schneider PR, Finger CAG, Fleig FD. (1992) Produção de madeira e casca de acácia-negra (Acacia mearnsii De Wild) em diferentes espaçamentos. Ciência Florestal, 2 (1): 87-97. doi: http://dx.doi.org/10.5902/19805098277.

Leite ES, Minette LJ, Fernandes HC, Souza AP, Amaral EJ, Lacerda EG. (2014). Desempenho do harvester na colheita de eucalipto em diferentes espaçamentos e declividades. Árvore, 38(1). doi: https://doi.org/10.1590/S010067622014000100009 .

Lima R, Inoue MT, Figueiredo Filho A, Araújo, AJ, Machado SA. (2013) Efeito do espaçamento no desenvolvimento volumétrico de Pinus taeda L. Floresta e Ambiente, 20 (2):223-230. doi: http://dx.doi.org/10.4322/floram.2013.001.

Melo LEL, Silva JRM, Napoli A, Lima JT, Trugilho PF, Nascimento DFR. (2016) Influence of genetic material and radial position on the anatomical structure and basic density of wood from Eucalyptus spp. and Corymbia citriodora. Scientia Forestalis, 44 (111): 611-621. doi: dx.doi.org/10.18671/scifor.v44n111.07.

Moulin JC, Arantes MDC, Oliveira JGL, Campinhos E, Gomes F, Vidaurre GB. (2017). Efeito do 
espaçamento, idade e irrigação no volume e densidade básica do eucalipto. Floresta e Ambiente, 24: e00073914. doi: https://dx.doi.org/10.1590/2179-8087.073914.

Müller MD, Couto L, Neves J L. (2005) Avaliação de um clone de eucalipto estabelecido em diferentes densidades de plantio para produção de biomassa e energia. Biomassa \& Energia, 2 (3): 177-186.

Oliveira JTS, Hellmeister JC, Tomazello Filho, M. (2005) Variação do teor de umidade e da densidade básica na madeira de sete espécies de eucalipto. Revista Árvore, 29 (1): 115-127. doi: http://dx.doi.org/10.1590/S0100-

67622005000100013 .

Oliveira Neto SN, Reis GG, Reis MGF, Leite HG, Neves JCL. (2003) Produção e distribuição de biomassa em Eucalyptus camaldulensis Dehn. em resposta adubação e ao espaçamento. Revista Árvore, 27 (1): 15-23.

Oliveira Neto SN, Reis GG, Reis MGF, Leite HG, Neves JCL. (2010) Crescimento e distribuição diamétrica de Eucalyptus camaldulensis em diferentes espaçamentos e níveis de adubação na região de cerrado de Minas Gerais. Floresta, 40 (4): 755-762. doi: http://dx.doi.org/10.5380/rf.v40i4.20327.

Oliveira TK, Macedo RLG, Venturin N, Higashikawa EM. (2009) Desempenho silvicultural e produtivo de eucalipto sob diferentes arranjos espaciais em sistema agrossilvipastoril. Pesquisa Florestal Brasileira, 1: 1-9. doi: http://dx.doi.org/10.4336/2009.pfb.60.01.

Passos CAM, Bufulim Junior L, Gonçalves MR. (2006) Avaliação silvicultural de Tectona grandis Lf, em Cáceres-MT, Brasil: resultados preliminares. Ciência Florestal, 16 (2): 225-232.

Reiner DA., Silveira ER., Szabo MS. (2011) O uso do eucalipto em diferentes espaçamentos como alternativa de renda e suprimento da pequena propriedade na região sudoeste do Paraná. Synergismus Scyentifica UTFPR, 6 (1).

Reis CAF, Santos PET, Paludzyszyn Filho E. (2014). Avaliação de clones de eucalipto em Ponta Porã, Mato Grosso do Sul. Pesquisa Florestal Brasileira, 34 (80): 263-269. doi: dx.doi.org/10.4336/2014.pfb.34.80.569.

Ribeiro MDSB, Blanco Jorge LA, Mischan MM, Santos AL, Ballarin AW. (2017) Avaliação da produção de biomassa do fuste de um clone híbrido de eucalipto sob diferentes espaçamentos. Ciência Florestal, 27 (1): 31-45. doi: http://dx.doi.org/10.5902/1980509826445.

Rocha MFV, Vital BR, Carneiro ACO, Carvalho AMML, Cardoso MT, Hein PRG. (2016) Effects of plant spacing on the physical, chemical and energy properties of Eucalyptus wood and bark. Journal of Tropical Forest Science, 28 (3): 243-248.

Santana WMS, Calegario N, Arantes MDC, Trugilho PF. (2012) Effect of age and diameter class on the properties of wood from clonal Eucalyptus. $\begin{array}{llll}\text { Cerne, } & 18 & \text { (1): } & 1-8 .\end{array}$ https://doi.org/10.1590/S010477602012000100001 .

Santiago FLS, Rezende MA. (2014) Aproveitamento de resíduos florestais de Eucalyptus spp. na indústria de fabricação de celulose para geração de energia térmica e elétrica. Energia na Agricultura, 29 (4): 241-253.

doi: https://doi.org/10.17224/EnergAgric.2014v29n4p24 $1-253$.

Sereghetti GC, Lanças KP, Sartori MS, Rezende MA, Soler RR. (2015) Efeito do espaçamento no crescimento e na densidade básica da madeira de Eucalyptus urophylla $x$ Eucalyptus grandis em florestas de ciclo curto. Energia na Agricultura, 30 (3): 257-262. doi: https://doi.org/10.17224/EnergAgric.2015v30n3p25 7-262.

Sereghetti GC, Lanças KP, Sartori MS, Rezende MA, Soler RR. (2015) Efeito do espaçamento no crescimento e na densidade básica da madeira de Eucalyptus urophylla x Eucalyptus grandis em florestas de ciclo curto. Energia na Agricultura, 30 (3): 257-262.

Silveira ER, Reiner DA, Smaniotto JR. (2014) Efeito do espaçamento de plantio na produção de madeira e serapilheira de Eucalyptus dunnii na região sudoeste do Paraná. Revista Técnico-Científica do CREA-PR, 2 (1): 1-9.

Soares CPB, Paula Neto FP, Souza AL. (2011) Dendrometria e inventário florestal. 2 ed. Viçosa: UFV. 272p.

Viera M, Schumacher MV, Caldeira MVW. (2013) Dinâmica de decomposição e nutrientes em plantio de Eucalyptus urophylla $\times$ Eucalyptus globulus no Sul do Brasil. Floresta e Ambiente, 20 (3): 351-360. doi: http://dx.doi.org/10.4322/floram.2013.021. 